\title{
Efectividad de la terapia antiretroviral en pacientes con VIH atendidos en el Hospital Verdi Cevallos Balda
}

\author{
Effectiveness of antiretroviral therapy in patients with HIV treated at the \\ Verdi Cevallos Balda Hospital
}

Cedeño Velez Juan ${ }^{1}$, Chiquito Alay Jocelyne ${ }^{2}$, Daza Mendoza Genessis ${ }^{3}$, Zambrano Centeno Heydee ${ }^{4}$, Palacios Cevallos Mauricio ${ }^{5}$ \& Cañarte Alcivar Jorge ${ }^{6}$

Recibido: 04-02-2019 / Revisado: 05-02-2019 /Aceptado: 05-03-2019/ Publicado: 04-04-2019

\begin{abstract}
DOI: https://doi.org/10.33262/cienciadigital.v9i2.373

The Human Immunodeficiency Virus (HIV) is a retrovirus of the lentivirus genus that causes Acquired Immune Deficiency Syndrome (AIDS), although mortality associated with HIV infection has decreased significantly as a result of universal access to antiretroviral therapy (ART). One of the main problems is the poor adherence to treatment for unknown reasons, which this research aims to identify with the effectiveness of antiretroviral therapy in HIV patients treated at the Verdi Cevallos Balda hospital.

The antiretroviral drugs break the schemes in which the (HIV / AIDS) was considered as a synonym of death, since, from terminal illness, it becomes a chronic disease with which it is possible to live together. However, antiretroviral treatment is not curative and must be administered for life. This means that factors such as the number of tablets, their tolerability, the toxicity of the treatment and the cost have a fundamental weight.
\end{abstract}

Keywords: Retroviruses, lentiviruses, Acquired Immune Deficiency Syndrome (AIDS), HIV infection, antiretroviral drugs.

\section{Resumen}

El Virus de la Inmunodeficiencia Humana (VIH) es un retrovirus del género lentivirus causante del Síndrome de Inmunodeficiencia Adquirida (SIDA), Si bien la mortalidad asociada a la infección por VIH ha disminuido significativamente

\footnotetext{
${ }^{1}$ Facultad de Ciencias de la Salud; Universidad Técnica de Manabí, jcedeno2887@utm.edu.ec

${ }^{2}$ Facultad de Ciencias de la Salud; Universidad Técnica de Manabí, jcedeno2887@utm.edu.ec

${ }^{3}$ Facultad de Ciencias de la Salud; Universidad Técnica de Manabí, jcedeno2887@utm.edu.ec

${ }^{4}$ Facultad de Ciencias de la Salud; Universidad Técnica de Manabí, jcedeno2887@utm.edu.ec

${ }^{5}$ Facultad de Ciencias de la Salud; Universidad Técnica de Manabí, Unidad de Atención Integral VIH SIDA; Hospital Verdi Cevallos Balda. jcedeno2887@utm.edu.ec

${ }^{6}$ Facultad de Ciencias de la Salud; Universidad Técnica de Manabí, jcedeno2887@utm.edu.ec
} 
producto del acceso universal a la terapia antirretroviral (TARV), uno de los principales problemas es la mala adherencia al tratamiento por razones desconocidas, que esta investigación pretende identificar con la efectividad de la terapia antiretroviral en pacientes con VIH atendidos en el hospital Verdi Cevallos Balda.

Los medicamentos antirretrovirales rompen los esquemas en el cual el (VIH/SIDA) era considerado como sinónimo de muerte, dado que, de enfermedad terminal, pasa a convertirse en una enfermedad crónica con la cual es posible convivir. Sin embargo, el tratamiento antirretroviral no es curativo y debe de ser administrado de por vida.

Palabras clave: Retrovirus, lentivirus, Síndrome de Inmunodeficiencia Adquirida (SIDA), infección por VIH, medicamentos antirretrovirales.

\section{Introducción.}

De acuerdo a publicaciones realizadas por la ONUSIDA fallecen cerca de 940.000 personas en todo el mundo a causa de enfermedades relacionadas con el sida, no obstante datos obtenidos por los mismos indican que el número de muertes descendió en comparación al 2004 y 2010 teniendo cifras de 1,9 millones y 1,4 millones tentativamente.

Esta es una disminución importante en el número de personas que fallecen cada año es producto al mayor acceso de terapias antirretrovirales la cuales han representado un antes y un después en la forma de tratamiento en la historia natural de la infección. A mediados de 2017, cerca de 20,9 millones de personas recibieron terapias antirretrovírica en todo el mundo. El TARGA o también conocido como HAART (highly active antiretroviral therapy) se refiere al conjunto de terapias antirretrovirales constituidas por tres o más fármacos, que permiten la supresión de replicación viral hasta niveles casi indetectables.

La efectividad es medida por parámetros clínicos y biológicos (carga viral y nivel de células CD4+, respectivamente), por ejemplo, el recuento de linfocitos CD4+ debe incrementarse a más de 200/mm3 con tratamiento antirretroviral durante un año, puesto que una persistente declinación de estos y un deterioro clínico de los pacientes, indica fallo del plan terapéutico.

El estudio pretende describir y conocer las características en pacientes con HIV a los que se aplica la terapia antirretroviral y su efectividad como también a los que se les aplica rescate en el hospital Verdi Cevallos Balda de Portoviejo.

Además, por esta razón, se propone establecer el porcentaje de pacientes cuyo tratamiento es efectivo, como también, el porcentaje de pacientes que tuviesen reportes de falla en su tratamiento, y su necesidad de cambio de esquema.

\section{Desarrollo}

El Virus de la Inmunodeficiencia Humana (VIH) es un retrovirus del género lentivirus causante del Síndrome de Inmunodeficiencia Adquirida (SIDA), descrito hace tres 
décadas. Se conocen dos subtipos: el VIH-1 y el VIH-2, siendo el primero el más común y de distribución mundial, mientras que el segundo es una variante menos virulenta, más prevalente en África Occidental y Central. ${ }^{11}$

El virión es esférico, dotado de una envoltura y con una cápside proteica. Su genoma es una cadena de ARN monocatenario que debe copiarse provisionalmente al ADN para poder multiplicarse e integrarse en el genoma de la célula que infecta. El proceso de conversión de ARN en ADN es una característica principal de los retrovirus y se lleva a cabo mediante acciones enzimáticas de transcriptasa inversa. Su parte exterior es la «cubierta», una membrana que originalmente pertenecía a la célula de donde el virus emergió. En la cubierta se encuentra una proteína del virus, la gp41, o «glicoproteína transmembrana». Conectada a la gp41 está la gp120, la cual puede unirse al receptor CD4 localizado en la superficie de los linfocitos T para penetrar en ellos. El núcleo tiene la «cápside», compuesta por la proteína p24. En su interior está el ARN, la forma de información genética del VIH. ${ }^{12}$

\section{Fases de la infección.}

Existen 3 fases de infección del VIH:

Infección aguda. - Dentro de los primeros días de la adquisición del VIH ocurre una enfermedad transitoria, a veces, sintomática asociada a altos niveles de replicación del VIH y a una rápida caída, por infección, de las células que tienen el receptor CD4+, la mayoría de ellas son linfocitos $\mathrm{T}$ CD4+ colaboradores y células dendríticas, especialmente las de Langerhans, y en minoría los macrófagos. ${ }^{13}$

Se define como infección aguda a la presencia de altos niveles de ARN viral en plasma en presencia de un Test de Elisa negativo y/o Western Blot negativos o indeterminados ( $<3$ bandas positivas) englobando respuesta inmune humoral.

Los test más sensibles y de cuarta generación pueden detectar a ambos, antígenos y anticuerpos, disminuyendo el "período de ventana" (virus positivo-anticuerpo negativo) en cinco días ${ }^{14}$. Los test para detección de RNA viral cierran esta diferencia por un adicional de siete días. ${ }^{11}$

Infección crónica. - Llamada infección asintomática por el VIH o fase de latencia clínica. El VIH sigue reproduciéndose en el cuerpo, pero en concentraciones muy bajas. Aunque personas con infección crónica por el VIH no tienen ningún síntoma relacionado con la misma, pueden propagar el virus a otras.

SIDA. - Es la fase final y más grave de la infección por el VIH. La infección disminuye gradualmente la cantidad de linfocitos T CD4+ en los tejidos y en la sangre, lo cual conduce al paciente a un estado grave de inmunosupresión celular tras el cual, el cuerpo no puede luchar contra las infecciones oportunistas y el cáncer. A las personas con el VIH se les diagnostica SIDA si tienen un recuento de linfocitos T CD4+ de menos de 200/mm3, o si presentan ciertas infecciones oportunistas. Sin tratamiento, por lo general, las personas con SIDA sobreviven unos 3 años. El estado de inmunosupresión es la consecuencia de la progresión de la infección del virus, sin que el paciente reciba 
tratamiento.

\section{Principales fármacos antirretrovirales.}

Inhibidores de la transcriptasa inversa: Constituyen la piedra angular del tratamiento antirretroviral. Incluyen los inhibidores de la transcriptasa inversa análogos de nucleósidos (ITIAN), e inhibidores de la transcriptasa inversa no análogos de nucleósidos (ITINAN).

Inhibidores de la transcriptasa inversa análogos de los nucleósidos (ITIAN): Actúan como sustratos alternativos para este enzima, impidiendo la retrotranscripción del RNA viral en DNA, interfiriendo así en el ciclo viral. Como contrapartida, estos fármacos son sustratos también de la DNA polimerasa $\gamma$ mitocondrial produciendo toxicidad a este nivel $[33,34]$.

Los principales fármacos de este grupo son Zidovudina (AZT), Didanosina (ddI), Estavudina (d4T), Lamivudina (3TC), Emtricitabina (FTC) y Abacavir (ABC). Se incluye en este grupo al Tenofovir (TDF), aunque éste último es un análogo de nucleótido.

El TDF, probablemente sea el fármaco antirretroviral más empleado en la actualidad. Se ha descrito toxicidad renal asociada, en algunos casos, tanto por daño tubular como Síndrome de Fanconi. También produce a largo plazo deterioro de la densidad mineral ósea.

Inhibidores de la transcriptasa inversa no análogos de nucleósidos. Estos fármacos, a diferencia de los análogos, no compiten con los nucleósidos naturales, sino que se unen al centro activo de la transcriptasa inversa, impidiendo así su actuación.

Son cuatro: Nevirapina (NVP), Efavirenz (EFV), Etravirina (ETR) y Rilpivirina (RPV). Estos fármacos producen habitualmente interacciones con otros por inducir algunas isoenzimas del citocromo p450.

La NVP, es un fármaco de amplia experiencia de uso. Produce clásicamente como efectos adversos, exantemas cutáneos, de tipo rash, en las primeras semanas y elevación de las transaminasas. Tiene importantes interacciones con otros fármacos porque induce el CYP 3 A4. No se recomienda en mujeres con CD4 por encima de $250 / \mu l$ ni en varones con cifras de CD4 por encima de $400 / \mu l$ por aumento de reacciones de hipersensibilidad en estas subpoblaciones.

La RPV produce menos efectos adversos que el EFV. En combinaciones de triple terapia con TDF/FTC, ha demostrado no inferioridad con respecto a EFV en grupos de pacientes con carga viral por debajo de 100.000 copias.

Finalmente, la ETR, produce menos efectos adversos que NVP y EFV y menos interacciones, pero su eficacia está menos contrastada.

Inhibidores de la proteasa: Los inhibidores de la proteasa (IP) son unos potentes antirretrovirales utilizados frecuentemente en combinación con dos ITIAN en terapias de inicio. Tienen una elevada barrera genética, que dificulta la selección de mutaciones de resistencia. Su mecanismo de acción se basa en la inhibición de la proteasa viral, lo que impide la fragmentación de los polipéptidos virales en proteínas que permitan constituir 
estructuras virales con capacidad infectiva.

Los IP potenciados disponibles son: Atazanavir (ATV), Darunavir (DRV), Lopinavir (LPV), Fosamprenavir (FPV), Saquinavir (SQV) y Tipranavir (TPV). Cobicistat, sólo está autorizado como potenciador de ATV y DRV y no tiene acción antirretroviral por sí mismo.

Con respecto a sus efectos adversos, aunque varían de intensidad según el IP, presentan fundamentalmente alteraciones gastrointestinales y son los de peor perfil metabólico.

Inhibidores de la integrasa: Esta nueva familia de fármacos antirretrovirales, tiene como mecanismo de acción la inhibición de la integrasa viral, que es la enzima responsable de la integración del DNA proviral en el genoma de la célula infectada.

Este proceso tiene varios pasos, y el inhibido por los fármacos es la transferencia de cadena, es decir la unión.

En diferentes ensayos clínicos se ha probado la eficacia y buena tolerabilidad de estos fármacos y al pertenecer a una familia distinta y tener diferente diana terapéutica no tienen resistencia cruzada con los demás fármacos.

Inhibidores de la entrada del VIH: Estos fármacos tienen como dianas terapéuticas, los diferentes pasos que se dan hasta la entrada del virus en la célula.

El primer paso es la unión de la gp120 con el receptor CD4 de la célula diana. Los fármacos que actúan en este primer paso son los inhibidores de la unión gp120-CD4. Al unirse ambas proteínas se produce un cambio conformacional en la gp120 que le permite unirse al correceptor CXCR4 o CCR5. Los fármacos que impiden este paso son los antagonistas CCR5 y de CXCR4.

Finalmente, la gp41viral presenta un cambio conformacional que permite la fusión de ambas membranas, produciéndose la entrada del virus. Los fármacos que impiden este paso final son los inhibidores de la fusión.

En la actualidad en el uso clínico diario se emplean fundamentalmente, los antagonistas de CCR5 donde el fármaco más estudiado es el Maraviroc (MVC) y los inhibidores de la fusión, como el enfuvirtide (ENF).

La efectividad de la terapia antiretroviral presenta resultados favorables en los pacientes con VIH atendidos en el hospital Verdi Cevallos Balda.

\section{Metodología.}

La Terapia antirretroviral (TARV) son medicamentos que tratan al VIH. Los medicamentos ni matan ni curan al virus, pero cuando tomado en combinación, pueden prevenir la reproducción del virus. A veces se llaman ARV. Se refiere a la terapia antirretroviral combinada como TARGA, de Gran Actividad. Los pacientes con VIH, en la actualidad cuentan, con medicamentos conocidos como los antirretrovirales, aquellos que le garantizan al paciente portador de este virus una oportunidad de poder manera el VIH, de una manera en que este pueda mantener un control de la carga viral presente en su organismo, y así mismo se encuentran los niveles de los linfocitos TCD4, presenten en 
el suero. Siendo este tratamiento muy beneficioso ya que, si el paciente se adapta a dicho tratamiento, este puede lograr una disminución considerable de la carga viral de dicho virus.

La presente investigación es descriptiva, retroprospectiva correlacional y con una secuencia longitudinal. realizada bajo el enfoque cuantitativo aquel que se basa en una forma estructurada de recopilar y analizar datos obtenidos de distintas fuentes, esta implica el uso de herramientas informáticas, estadísticas, y matemáticas para obtener resultados, para posteriormente llevar a cabo el análisis de los datos, para de esta manera comprobar la hipótesis establecida previamente, es concluyente en su propósito ya que trata de cuantificar el problema y entender qué tan generalizado está mediante la búsqueda de resultados proyectarles a una población mayor. Siendo estos datos obtenidos de la fuente de datos del Hospital Verdi Cevallos Balda.

La muestra estuvo conformada por 200 pacientes, cuyos criterios de inclusión son Pacientes con VIH, tratados en el Hospital Verdi Cevallos Balda y que se encuentren, con el tratamiento de Terapia Antirretroviral (Triterapia y Biterapia)- de los cuales hasta el momento se ha recopilado información de 18 pacientes, pero disminuyendo a 15 pacientes, ya que tres de ellos no cuentan con valores de carga viral y TCD4. La población seleccionada para la presente investigación está conformada, por los pacientes con VIH, excluyendo a los Pacientes que no se apliquen el tratamiento dentro del Hospital Verdi Cevallos Balda y a los pacientes que no reflejan datos de carga viral ni CD4 para la presente investigación.

\section{Análisis e interpretación de resultados.}

\section{Tratamientos generales:}

\begin{tabular}{|l|c|c|}
\hline \multicolumn{3}{|c|}{ Tabla \#1 } \\
\hline ABC/3TC+LPV/RTV & 1 & $4 \%$ \\
\hline AZT/3TC+EFV & 2 & $7 \%$ \\
\hline AZT/3TC+LPV/RTV & 1 & $4 \%$ \\
\hline RAL+DRV+RTV & 1 & $4 \%$ \\
\hline RAL+DRV+RTV+TDF+FTC & 1 & $4 \%$ \\
\hline TDF/FTC/EFC & 0 & $0 \%$ \\
\hline TDF/FTC/EFV & 16 & $57 \%$ \\
\hline TDF/FTC/RAL & 2 & $7 \%$ \\
\hline TDF/FTC+ DRV+ RTV & 3 & $11 \%$ \\
\hline TDF/FTC+RAL+LPV/RTV & 1 & $4 \%$ \\
\hline TOTAL & 28 & $100 \%$ \\
\hline
\end{tabular}

En base a los datos estadísticos generales y exhaustivos se evidencia que de entre los datos de 28 usuarios los tratamientos con medicamentos para el control de HVI más 
empleados es el TDF/FTC/EFV con una frecuencia de 16 usuarios que representa un 57\%; $\mathrm{y}$ en menor aplicación los tratamientos con medicamentos AZT/3TC+EFV y TDF/FTC/RAL con un 7\%, seguido de TDF/FTC+ DRV+ RTV con un $11 \%$, como finalmente en mucho menos proporción la aplicación de terapias con fármacos ABC/3TC+LPV/RTV, AZT/3TC+LPV/RTV,

RAL+DRV+RTV, RAL+DRV+RTV+TDF+FTC y TDF/FTC+RAL+LPV/RTV en un 4\%. A partir del análisis se establece que entre la mayoría del total de usuarios en tratamiento con cada uno de los medicamentos y basándose en su frecuencia de uso podemos interpretar que el TDF/FTC/EFV en apariencia resulta contribuir más a la evolución psitiva de los pacientes.

\section{Pacientes actuales con los tratamientos:}

\begin{tabular}{|l|c|c|}
\hline \multicolumn{3}{|c|}{ Tabla\# 2 } \\
\hline ABC/3TC+LPV/RTV & 1 & $6 \%$ \\
\hline AZT/3TC+EFV & 0 & $0 \%$ \\
\hline AZT/3TC+LPV/RTV & 0 & $0 \%$ \\
\hline RAL+DRV+RTV & 0 & $0 \%$ \\
\hline RAL+DRV+RTV+TDF+FTC & 1 & $6 \%$ \\
\hline TDF/FTC/EFC & 0 & $0 \%$ \\
\hline TDF/FTC/EFV & 12 & $67 \%$ \\
\hline TDF/FTC/RAL & 1 & $6 \%$ \\
\hline TDF/FTC+ DRV+ RTV & 2 & $11 \%$ \\
\hline TDF/FTC+RAL+LPV/RTV & 1 & $6 \%$ \\
\hline TOTAL & 18 & $100 \%$ \\
\hline
\end{tabular}

Tomando en cuenta los datos estadísticos se puede apreciar que los pacientes que se encuentran en actual tratamiento 12 del total de la población en estudio recibe tratamiento con TDF/FTC/EFV representando un porcentaje de casi el $67 \%$ del total de usuarios y en menores proporciones los tratamientos con TDF/FTC+ DRV+ RTV en un 11\%, seguido de ABC/3TC+LPV/RTV, TDF/FTC/RAL y TDF/FTC+RAL+LPV/RTV en un 6\%. De acuerdo a lo analizado se obtuvieron datos de 18 pacientes con diferentes tratamientos en los que se puede evidenciar el predominio de los tratamientos con TDF/FTC/EFV, sin embargo, para previsar un estudio más eficaz 3 pacientes fueron excluidos del estudio por los siguientes motivos: 2 de ellos presentaban tan sólo un unico valor de CV como de CD4 lo que no permite conocer si el usuario mostraba mejoría o no; en cuanto al tercero no disponía de datos de CV ni CD4 para su último tratamiento, razón la cual solo 15 individuos fueron tomados como muestra para el presente estudio. Los datos pueden tener margen de error desconocido ya que al comenzar o cambiar de tratamiento de se conocen los niveles de CV o CD4 de ese día específico, ya que la diferencia de fechas puede ser de hasta meses.

\section{Tratamientos útiles para estudio:}




\begin{tabular}{|l|c|c|}
\hline \multicolumn{3}{|c|}{ Tabla \#3 } \\
\hline ABC/3TC+LPV/RTV & 1 & $7 \%$ \\
\hline AZT/3TC+EFV & 0 & $0 \%$ \\
\hline AZT/3TC+LPV/RTV & 0 & $0 \%$ \\
\hline RAL+DRV+RTV & 0 & $0 \%$ \\
\hline RAL+DRV+RTV+TDF+FTC & 1 & $7 \%$ \\
\hline TDF/FTC/EFC & 0 & $0 \%$ \\
\hline TDF/FTC/EFV & 9 & $60 \%$ \\
\hline TDF/FTC/RAL & 1 & $7 \%$ \\
\hline TDF/FTC+ DRV+RTV & 2 & $13 \%$ \\
\hline TDF/FTC+RAL+LPV/RTV & 1 & $7 \%$ \\
\hline TOTAL & 15 & $100 \%$ \\
\hline
\end{tabular}

De acuerdo con los marcadores porcentuales extra se manifiesta que en mayor proporción el tratamiento con TDF/FTC/EFV representado con un $60 \%$ a la frecuencia de 9 personas manteniendo que en los tratamientos el TDF/FTC/EFV es el más administrado, seguido en menores proporciones los tratamientos con TDF/FTC+ DRV+ RTV que sólo se vió administrado a 2 pacientes lo que refleja un 13\%; en cuanto a los últimos también empleados como ABC/3TC+LPV/RTV, RAL+DRV+RTV+TDF+FTC, TDF/FTC/RAL y TDF/ FTC+RAL+LPV/RTV que respectivamente equivalen a un $7 \%$ de entre los aplicados, demostrando, además, que los tratamientos restantes existen, pero son menos empleados. Mediante lo ya expresado se determina que entre todos los tratamientos a disposición de los 15 pacientes seleccionados el estudio los medicamentos $\mathrm{TDF} / \mathrm{FTC} / \mathrm{EFV}$ son los más empleados para tratar la terapia contra el virus.

\section{Eficacia de la Carga Viral (CV).}

Tabla 4.

\begin{tabular}{|l|l|l|}
\hline Tratamientos & & $\sum$ Eficacia CV \\
\hline ABC/3TC+LPV/RTV & 1 & 0,010989011 \\
\hline AZT/3TC+EFV & 0 & \\
\hline AZT/3TC+LPV/RTV & 0 & \\
\hline RAL+DRV+RTV & 0 & \\
\hline RAL+DRV+RTV+TDF+FTC & 1 & $-0,036630037$ \\
\hline TDF/FTC/EFC & 0 & \\
\hline TDF/FTC/EFV & 9 & 303,8992919 \\
\hline TDF/FTC/RAL & 1 & 7,388888889 \\
\hline TDF/FTC+ DRV+ RTV & 2 & $-0,037293057$ \\
\hline
\end{tabular}




\begin{tabular}{|l|c|l|} 
TDF/FTC+RAL+LPV/RTV & 1 & $-115,2057762$ \\
\hline TOTAL & 15 & \\
\hline
\end{tabular}

Eficacia de los CD4.

Tabla 5.

\begin{tabular}{|l|c|c|}
\hline Tratamientos útiles para estudio: & & Eficacia CD4 \\
\hline ABC/3TC+LPV/RTV & 1 & 0,03021978 \\
\hline AZT/3TC+EFV & 0 & \\
\hline AZT/3TC+LPV/RTV & 0 & \\
\hline RAL+DRV+RTV & 0 & \\
\hline RAL+DRV+RTV+TDF+FTC & 1 & $-0,032967033$ \\
\hline TDF/FTC/EFC & 0 & \\
\hline TDF/FTC/EFV & 9 & 0,734050502 \\
\hline TDF/FTC/RAL & 1 & 0,189260563 \\
\hline TDF/FTC+ DRV+RTV & 2 & 0,009393285 \\
\hline TDF/FTC+RAL+LPV/RTV & 1 & 0,09566787 \\
\hline TOTAL & 15 & \\
\hline
\end{tabular}

En los datos expuestos se observa la "Eficacia tratamiento CV" que representa la C, el cual disminuye por día de tratamiento desde el día del inicio del tratamiento hasta el día de recolección de datos o cambio de tratamiento, este valor es para un tratamiento específico. Este valor es para todos los tratamientos en conjunto del paciente.

Los marcadores expuestos en cuanto a la "Eficacia tratamiento CD4" que representa los CD4 que aumentan por día de tratamiento desde el día del inicio del tratamiento hasta el día de recolección de datos o cambio de tratamiento, este valor es para un tratamiento específico. Este valor es para todos los tratamientos en conjunto del paciente.

\section{Media de la Carga Viral (CV).}

Tabla 6.

\begin{tabular}{|l|c|c|}
\hline Tratamientos útiles para estudio: & & Media CV \\
\hline ABC/3TC+LPV/RTV & 1 & 0,010989011 \\
\hline AZT/3TC+EFV & 0 & \\
\hline AZT/3TC+LPV/RTV & 0 & \\
\hline RAL+DRV+RTV & 0 & \\
\hline RAL+DRV+RTV+TDF+FTC & 1 & $-0,036630037$ \\
\hline TDF/FTC/EFC & 0 & \\
\hline TDF/FTC/EFV & 9 & 33,76658799 \\
\hline TDF/FTC/RAL & 1 & 7,388888889 \\
\hline TDF/FTC+ DRV+ RTV & 2 & $-0,018646529$ \\
\hline
\end{tabular}




\begin{tabular}{|l|c|l|} 
TDF/FTC+RAL+LPV/RTV & 1 & $-115,2057762$ \\
\hline TOTAL & 15 & \\
\hline
\end{tabular}

Media de los CD4.

Tabla 7.

\begin{tabular}{|l|c|c|}
\hline Tratamientos útiles para estudio: & & Media CD4 \\
\hline ABC/3TC+LPV/RTV & 1 & 0,03021978 \\
\hline AZT/3TC+EFV & 0 & \\
\hline AZT/3TC+LPV/RTV & 0 & \\
\hline RAL+DRV+RTV & 0 & \\
\hline RAL+DRV+RTV+TDF+FTC & 1 & $-0,032967033$ \\
\hline TDF/FTC/EFC & 0 & \\
\hline TDF/FTC/EFV & 9 & 0,081561167 \\
\hline TDF/FTC/RAL & 1 & 0,189260563 \\
\hline TDF/FTC+ DRV+RTV & 2 & 0,004696643 \\
\hline TDF/FTC+RAL+LPV/RTV & 1 & 0,09566787 \\
\hline TOTAL & 15 & \\
\hline
\end{tabular}

Desviación de la Carga Viral (CV).

Tabla 8.

\begin{tabular}{|l|c|c|}
\hline Tratamientos útiles para estudio: & & Desviación CV \\
\hline ABC/3TC+LPV/RTV & 1 & 0 \\
\hline AZT/3TC+EFV & 0 & \\
\hline AZT/3TC+LPV/RTV & 0 & \\
\hline RAL+DRV+RTV & 0 & \\
\hline RAL+DRV+RTV+TDF+FTC & 1 & 0 \\
\hline TDF/FTC/EFC & 0 & \\
\hline TDF/FTC/EFV & 9 & 76,14544202 \\
\hline TDF/FTC/RAL & 1 & 0 \\
\hline TDF/FTC+ DRV+ RTV & 2 & 0,068762666 \\
\hline TDF/FTC+RAL+LPV/RTV & 1 & 0 \\
\hline TOTAL & 15 & \\
\hline
\end{tabular}

Desviación de la CD4.

\begin{tabular}{|l|c|c|}
\hline Tratamientos útilies para estudio: & & Desviación CD4 \\
\hline ABC/3TC+LPV/RTV & 1 & 0 \\
\hline AZT/3TC+EFV & 0 & \\
\hline AZT/3TC+LPV/RTV & 0 & \\
\hline RAL+DRV+RTV & 0 & \\
\hline
\end{tabular}




\begin{tabular}{|l|c|c|} 
RAL+DRV+RTV+TDF+FTC & 1 & 0 \\
\hline TDF/FTC/EFC & 0 & \\
\hline TDF/FTC/EFV & 9 & 0,086897921 \\
\hline TDF/FTC/RAL & 1 & 0 \\
\hline TDF/FTC+ DRV+ RTV & 2 & 0,24163721 \\
\hline TDF/FTC+RAL+LPV/RTV & 1 & 0 \\
\hline TOTAL & 15 & \\
\hline
\end{tabular}

\section{Conclusiones.}

- De acuerdo a lo esquematizado en razón de la eficacia y datos estadísticos en función de las Cargas Virales (CV) y CD4 vinculado a los tratamientos con las diferentes terapias medicamentosas para el control del HVI se establecen los siguientes criterios:

- Tratamiento con: ABC/3TC+LPV/RTV

Existe un paciente con este tratamiento, el cual estuvo primero con $\mathrm{TDF} / \mathrm{FTC} / \mathrm{EFV}$, este tratamiento tiene una eficacia de CV de 0.011 y una eficacia de CD4 de 0.030 en un periodo de 728 días.

- Tratamiento con: AZT/3TC+EFV

Existen 2 pacientes que tuvieron este tratamiento el cual fue reemplazado en los dos casos por TDF/FTC/EFV.

En el primer paciente tuvo una eficacia de CV desconocida y una eficacia de CD4 de 0.178 durante un periodo de 623 días después fue cambiado el tratamiento.

El segundo paciente tuvo una eficacia de CV desconocida y una eficacia de CD4 de 0.006 durante un periodo de 3198 días.

\section{- Tratamiento con: AZT/3TC+LPV/RTV}

Existe un paciente que tuvo este tratamiento, el cual fue reemplazado por RAL+DRV+RTV, tuvo una eficacia CV de -6.662 y una eficacia CD4 de -0.006 durante 1000 días.

\section{- Tratamiento con: RAL+DRV+RTV}

Existe un paciente que tuvo este tratamiento, el cual fue cambiado por RAL+DRV+RTV+TDF+FTC, este presentó una eficacia de CV desconocida al igual que la eficacia de CD4 durante 185 días.

- Tratamiento con: RAL+DRV+RTV+TDF+FTC

Existe un paciente con este tratamiento actualmente, el cual tiene una eficacia de CV de -0.037 y una eficacia de CD4 de -0.033 durante 273 días.

\section{- Tratamiento con: TDF/FTC/EFV}

Existen 16 pacientes que estuvieron o están con TDF/FTC/EFV 
El primer paciente tiene una eficacia de CV 0 y una eficacia de CD4 de 0.333 durante 18 días, hasta cambiar a TDF/FTC/RAL.

El segundo paciente tiene una eficacia de CV -70.875 y una eficacia de CD4 de 0.809 durante 529 días, hasta cambiar a TDF/FTC+ DRV+ RTV.

El tercer paciente tiene una eficacia de CV 352.566 y una eficacia de CD4 de 0.010 durante 926 días, hasta cambiar a ABC/3TC+LPV/RTV.

El cuarto paciente tiene una eficacia de CV -296.648 y una eficacia de CD4 de 0.349 durante 651 días, hasta cambiar a TDF/FTC+RAL.

Pacientes que están con este tratamiento

El primer paciente tiene una eficacia de CV de -0.024 y una eficacia de CD4 de 0.019 durante 1611 días.

El segundo paciente tiene una eficacia de CV de 36.627 y una eficacia de CD4 de -0.075 durante 654 días.

El tercer paciente tiene una eficacia de $\mathrm{CV}$ desconocida al igual que su eficacia de CD4 durante 261 días.

El cuarto paciente tiene una eficacia de $\mathrm{CV}$ desconocida al igual que su eficacia de CD4 durante 168 días.

El quinto paciente tiene una eficacia de CV de -0.023 y una eficacia de CD4 de 0.164 durante 1664 días.

El sexto paciente tiene una eficacia de CV de -0.024 y una eficacia de CD4 de 0.199 durante 1635 días.

El séptimo paciente tiene una eficacia de $\mathrm{CV}$ desconocida al igual que la eficacia de CD4 durante 293 días.

El octavo paciente tiene una eficacia de CV de 233.803 y una eficacia de CD4 de 0.010 durante 1012 días.

El noveno paciente tiene una eficacia de CV de 23.179 y una eficacia de CD4 de 0.147 durante 910 días.

El décimo paciente tiene una eficacia de CV de 10.189 y una eficacia de CD4 de 0.070 durante 572 días.

El onceavo paciente tiene una eficacia de CV de 0.203 y una eficacia de CD4 de 0.122 durante 572 días.

El doceavo paciente tiene una eficacia de CV de -0.030 y una eficacia de CD4 de 0.077 durante 1291 días.

- Tratamiento con: TDF/FTC/RAL

Existe un paciente que tuvo este tratamiento, el cual fue reemplazado por TDF/FTC+DRV+RTV, tenía una eficacia de CV de -0.218 y una eficacia de CD4 
de 5.472 durante 55 días.

Existe un paciente que tiene este tratamiento tiene una eficacia de CV de $7.389 \mathrm{y}$ una eficacia de CD4 de 0.189 durante 1136 días.

- Tratamiento con: TDF/FTC+ DRV+ RTV

Existe un paciente que tuvo este tratamiento, el cual fue reemplazado por TDF/FTC+RAL+LPV/RTV, tenía una eficacia de CV de 41.202 y una eficacia de CD4 de -0.089 durante 436 días.

Existen dos pacientes que tienen este tratamiento

El primer paciente tiene una eficacia de CV de 0.030 y una eficacia de CD4 de 0.175 durante 834 días.

El segundo paciente tiene una eficacia de CV de -0.067 y una eficacia de CD4 de -0.167 durante 996 días.

\section{- Tratamiento con: TDF/FTC+RAL+LPV/RTV}

Existe un paciente que tiene este tratamiento el cual posee una eficacia de $\mathrm{CV}$ de -115.205 y una eficacia de CD4 de 0.096 durante 554 días.

En cuanto a lo interpretado se puede plantear que el tratamiento que tiene una mejor eficacia media de CV es el tratamiento de TDF/FTC/EFV con 33.766.

El tratamiento que tiene una mejor eficacia media de CD4 es el tratamiento de TDF/FTC/RAL con 0.190 .

El tratamiento que más rápido hace llegar a niveles indetectables es el de ABC/3TC+LPV/RTV con 728 días.

- Conflicto de intereses: los autores declaran no tener conflicto de intereses existentes.

\section{Referencias Bibliográficas}

Unida, O. d. (2 de noviembre de 2018). ONUSIDA. Obtenido de ONUSIDA: http://www.unaids.org/es/resources/fact-sheet.

González Tomé, M., Ramos Amador, J., Guillén, S., Rojo, P., \& Ruiz Contreras, J. (2005). Efectividad de la terapia antirretroviral en niños con infección por VIH-1. Corte transversal. ANALES DE PEDIATRÍA, 1-102.

Rodríguez Alfaro, Maryenis, Gross Fernández, Caridad, \& Casas Gross, Sandra. (2011). Efectividad del tratamiento antirretroviral en pacientes con virus de inmunodeficiencia humana/sida en un hospital de Zimbabwe. MEDISAN, 15(4), 433-441. Recuperado en 03 de noviembre de 2018, de http://scielo.sld.cu/scielo.php?script=sci_arttext\&pid=S10293019201100040000 
$5 \& \operatorname{lng}=\mathrm{es} \& \ln g=\mathrm{es}$

Organización Mundial de la Salud. Un vistazo al Tratamiento 2.0. ONUSIDA [Internet]. 2012;3. Available from: http://www.who.int/hiv/pub/arv/treatment2_flyer_es.pdf?ua=1 Organización Panamericana de la Salud. Ecuador. Tratamiento antirretroviral bajo la lupa: un análisis de salud pública en Latinoamérica y el Caribe [Internet]. 2012; Available from: https://www.paho.org/hq/dmdocuments/2012/TAR-HojaEcuador.pdf Redacción MSF. Ecuador: traspaso del tratamiento con antirretrovirales al Plan Nacional de SIDA [Internet]. Médicos sin Fronteras. 2006 [cited 2018 Nov 3]. Available from: $\quad$ https://www.msf.es/actualidad/ecuador-traspaso-del-tratamientoantirretrovirales-al-plannacional-sida

Ministerio de Salud Pública Ecuador. Ecuador presentó el Plan Estratégico Multisectorial para la respuesta al VIH 2018-2022 [Internet]. Ministerio de Salud Pública Ecuador. 2018 [cited 2018 Nov 2]. Available from: https://www.salud.gob.ec/ecuador-presento-el-planestrategico-multisectorialpara-la-respuesta-al-vih-2018-2022/

Ministerio de Salud Pública Ecuador. Antirretrovirales para pacientes con VIH se distribuyen con normalidad en todo el país [Internet]. Ministerio de Salud Pública Ecuador. 2012. Available from: https://www.salud.gob.ec/antirretroviralesparapacientes-con-vih-se-distribuyen-con-normalidad-en-todo-el-pais/\#

Redacción El Tiempo. Veeduría vigila atención a las pacientes con VIH. El Tiempo [Internet]. $2017 \quad$ Nov $\quad 7 ; \quad$ Available from: https://www.eltiempo.com.ec/noticias/ecuador/4/veeduria-vigila-atencion-alaspacientes-con-vih

Redacción El Diario. 98 personas tienen VIH en Manabí y no lo saben. El Diario [Internet]. 2013 Dec 1; Available from: http://www.eldiario.ec/noticiasmanabiecuador/295336-98-personas-tienen-vih-en-manabi-y-no-lo-saben/

S.E. C. VIH: Infección aguda, pesquisa y manejo. Revista Médica Clínica Las Condes. $2014 ; 3(25)$.

Chávez Rodríguez CM. Revisión Bibliográfica sobre VIH/sida. Revista Médica MULTIMED. 2013; 4(17).

U TG. Infección por VIH y SIDA, dos mundos que se apartan. Revista de la Facultad de Medicina de la UNAM. 2016; 3(15).

Departamento de Salud y Servicios Humanos de EE.UU. InfoSIDA. [Online]; 2018. 


Acceso 2 de noviembre de 2018. Disponible en:

https://infosida.nih.gov/understandinghiv-aids/fact-sheets/19/46/las-fases-de-lainfeccion-por-el-vih.

FAÍLDE GARRIDO J.M LCJ. Influencia de las variables clínico-biológicas CD4 y carga viral sobre el rendimiento neuropsicológico de pacientes con infección por VIH1.

ANALES DE MEDICINA INTERNA. 2005; 22(6).

Somarriba Moreno J. Estrategias de simplificación del tratamiento antirretroviral en España. Eficacia clínica y virológica.

https://www.aidsmap.com/v634327617390300000/file/1050788/hiv_therapy_SPA.pdf.

[Online].

http://www.bibliotecaminsal.cl/wp/wp-content/uploads/2016/01/Las-

terapiasantiretrovirales-en-la-infecci\%C3\%B3n-por-VIH.pdf. [Online].

https://www.msdmanuals.com/es-ec/professional/enfermedades-infecciosas/virus-

deinmunodeficiencia-humana-hiv/infecci\%C3\%B3n-por-el-virus-deinmunodeficienciahumana-hiv. [Online].

https://www.msdmanuals.com/es-ec/professional/enfermedades-infecciosas/virus-

deinmunodeficiencia-humana-hiv/infecci\%C3\%B3n-por-el-virus-deinmunodeficienciahumana-hiv. [Online].

Yunquera-romero L. Dual therapy as an alternative treatment in HIV pretreatred patients: experience in a tertiary hospital. 2016; 29(1):25-31.

GESIDA. Documento de concenso GESIDA/Plan nacional sobre el Sida respecto al tratamiento antirretroviral en adultos con infeccion por le virus de inmunodeficiencia humana. 2015. 2015;

Batanero C. Situación Actual Y Perspectivas Futuras De La Educación Estadística. 1998; gTt-VIH | AIDS 2018: La biterapia con dolutegravir y lamivudina sería eficaz en personas con el VIH sin experiencia en tratamientos [Internet]. Gtt-vih.org. 2018 [cited 2

November 2018]. Available from: http://gtt-vih.org/actualizate/la_noticia_del_dia/260718

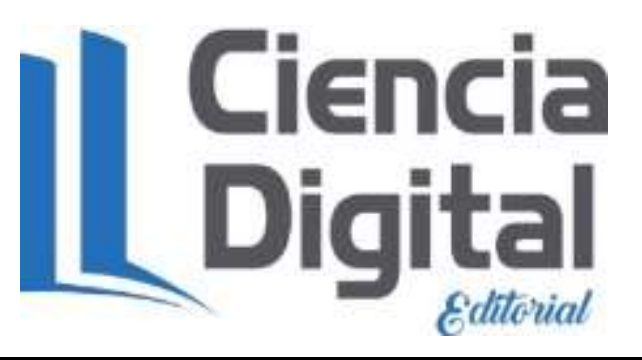


Para citar el artículo indexado.

Cedeño J., Chiquito J., Daza G., Zambrano H., Palacios M., \& Cañarte J. (2019) Efectividad de la terapia antiretroviral en pacientes con VIH atendidos en el Hospital Verdi Cevallos Balda. Revista electrónica Ciencia Digital 3(2), 27 - 42. Recuperado desde:

http://cienciadigital.org/revistacienciadigital2/index.php/CienciaDigital/article/view/373 $\underline{1818}$

\section{LC Ciencia}

El artículo que se publica es de exclusiva responsabilidad de los autores y no necesariamente reflejan el pensamiento de la Revista Ciencia Digital.

El artículo queda en propiedad de la revista y, por tanto, su publicación parcial y/o total en otro medio tiene que ser autorizado por el director de la Revista Ciencia Digital.
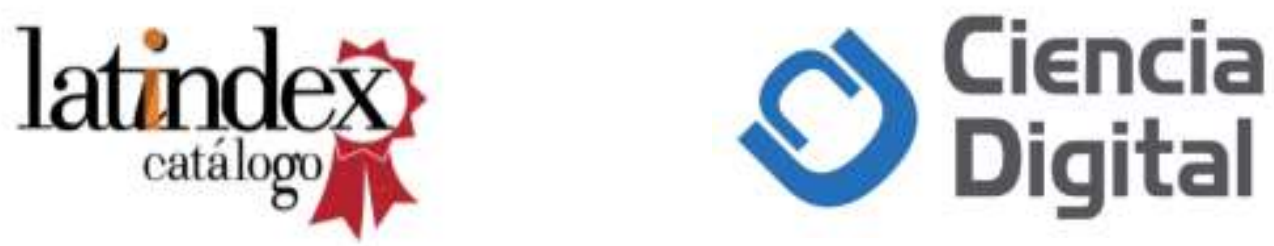